\title{
Gravitational spin-orbit interaction: criteria of increase
}

\author{
R.Plyatsko \\ Pidstryhach Institute for Applied Problems of Mechanics and Mathematics \\ of the National Academy of Sciences of Ukraine \\ $3^{6}$ Naukova St., UA-290601 Lviv, Ukraine
}

Received March 10, 1998

The gravitational spin-orbit interaction in the region of the ultrarelativistic orbital velocity of a spinning test particle in the Schwarzschild field is investigated on the basis of the Mathisson-Papapetrou equations. Different indications of an essential increase of this interaction, when the tangential velocity becomes ultrarelativistic, are analysed. The main factor of growth is the square of the relativistic Lorentz $\gamma$-factor. Numerical estimations for a high-energy electron in its path near the Earth's surface and near a neutron star are given. It is stressed that the widely recognised assumption that the deviation of a spinning test particle from the geodesic trajectory is caused by tidal forces is not correct. The real reason for this deviation is the gravitational spin-orbit interaction.

Key words: Mathisson-Papapetrou equations, ultrarelativistic motions

PACS: 04.20.-q, 95.30.Sf, 98.80.Dr

\section{Introduction}

The motion of a spinning test particle in a gravitational field differs from the geodesic motion. The corresponding equations were obtained by M.Mathisson and A.Papapetrou [1,2] (see, e.g., [3] concerning the history of the Mathisson-Papapetrou eq. (MPE) investigation). In [4] the physical reason for the deviation of a spinning test particle from the geodesic trajectory was touched. Namely, for motions in a Schwarzschild field "... a sort of interaction energy describing a spin-orbit coupling" [4], p. 262 was pointed out. A more detailed analysis of the gravitational spin-orbit (and spin-spin) interaction was made in [5]. At the same time another version on the nature of forces that deflect the motion of a spinning test particle from the corresponding motion of a test particle without a spin is present in some publications. For example, in [6] one can read that the gradient of a gravitational field causes a nongeodesic motion of a spinning test particle (see the footnote on page 18 of [6]). We find the analogous conclusion in the known work [7], exercise 
40.8 where tidal forces are discussed in the context of the MPE. After [6,7] this version was repeated in $[8,9]$. In particular, in [8], p. 417 "... in a curved spacetime spinning test particles do not follow geodesics because in relativity a spinning particle has to have a finite extension so that it feels tidal forces".

The correct identification of forces deviating a spinning test particle from the geodesic trajectory is very important for the prediction of physical consequences of the MPE. If one supposes that these forces have the tidal nature, then the spin of a test particle is able only slightly to deform the world line and there are only two situations where the tidal forces might become important: for rapidly spinning neutron stars and for electron outside a body at the late stage of a gravitational collapse [7], p. 1121.

However, in $[10,11]$ we showed that the widely recognized assumption as to the tidal forces as the reason for the deviation of a spinning test particle from the geodesic trajectory is not correct. This fact is a direct consequence of the MPE in the frame of reference co-moving with a spinning test particle. We can also indicate two other arguments in support of the conclusion that the MPE do not contain tidal forces. For example, let us assume that tidal forces are present in the MPE. Then, these forces cannot disappear if we make the spin equal to zero (more exactly, the angular velocity of the inner rotation) in the MPE, because the tidal forces are connected with the dimension of a test particle and its nonrotating state does not remove them. However, if one puts a spin equal to 0, the MPE transform into geodesic equations, but not into equations of geodesic deviation. The geodesic equations do not contain tidal forces (in contrast to geodesic deviation ones, which do) and, therefore, the assumption that tidal forces are present in the MPE is not correct. It should be remembered that the tidal forces will be taken into account if we consider two close world lines (see [12], chapter 6, section 10, where a clear procedure for the derivation of geodesic deviation equations is given). However, the MPE, as well as geodesic equations, describe only one world line. Therefore, we can point out the place in the procedure of the MPE derivation where the tidal forces were neglected: when the world tube of a test particle was replaced with only one world line (see, e.g., [2], p. 250).

The second additional argument, which refutes the assumption that the presence of tidal forces is the reason for the deviation of a spinning test particle from the geodesic motion, is connected with the known fact that the MPE are the classical limit of the general relativistic Dirac equation [8]. Obviously, one cannot speak about tidal forces in the Dirac equation.

We stress: the authors of [7] do not provide a proof of the statement that tidal forces are present in the MPE; it is an assumption (hypothesis) only. In all probability, this assumption was caused by a too wide and universal interpretation of the weak equivalence principle in general relativity [10].

So, our investigation of the MPE demonstrates that in accordance with these equations there is a sole reason for the deviation of a spinning test particle from the geodesic trajectory in the Schwarzschild field, namely, the gravitational spinorbit interaction $[3,10,11]$. Here we shall consider the circumstances under which 
this interaction increases and can essentially influence the particle motion.

\section{The mathematical criterion}

When written in terms of the 4-vector velocity of the spinning particle $u^{\lambda}$ and its intrinsic angular momentum tensor $S^{\mu \nu}$, the MPE take the form $[1,2]$

$$
\begin{gathered}
\frac{\mathrm{D}}{\mathrm{d} s}\left(M u^{\lambda}+u_{\mu} \frac{\mathrm{D} S^{\lambda \mu}}{\mathrm{d} s}\right)=-\frac{1}{2} u^{\pi} S^{\rho \sigma} R_{\pi \rho \sigma}^{\lambda}, \\
\frac{\mathrm{D} S^{\mu \nu}}{\mathrm{d} s}+u^{\mu} u_{\sigma} \frac{\mathrm{D} S^{\nu \sigma}}{\mathrm{d} s}-u^{\nu} u_{\sigma} \frac{\mathrm{D} S^{\mu \sigma}}{\mathrm{d} s}=0,
\end{gathered}
$$

where $M$ is a function depending on the particle mass; $R_{\pi \rho \sigma}^{\lambda}$ is a curvature tensor; $\mathrm{D} / \mathrm{d} s$ is a symbol of the covariant derivative with respect to the velocity vector derivation. The set of equations (1), (2) is incomplete. This incompleteness reflects the physical assumptions made while obtaining (1), (2): these equations were derived without specifying any point inside the extended particle whose motion characterizes the motion of the particle as a whole. A natural desire to describe the mass center motion of a spinning particle leads to adding one more system which consists of three independent relations $[1,13,14]$

$$
S^{\mu \nu} u_{\nu}=0
$$

Equations (3) are called Pirani's additional condition. The number of equations in systems (1)-(3), with $u_{\mu} u^{\mu}=1$ taken into account, coincides with the number of unknown functions, $M$ being an integral of motion. Equation (1) with condition (3) can be rewritten as

$$
M \frac{\mathrm{D} u^{\lambda}}{\mathrm{d} s}-S^{\lambda \mu} \frac{\mathrm{D}^{2} u_{\mu}}{\mathrm{d} s^{2}}=-\frac{1}{2} u^{\pi} S^{\rho \sigma} R_{\pi \rho \sigma}^{\lambda} .
$$

That is, the second derivatives from the velocity appear in equations (1) due to (3). Therefore, prescribing in equations (1)-(3) the initial values of coordinates velocity and spin only, we cannot be sure that we will obtain more than one solution of a complete system of the MPE. Indeed, equations (1)-(3) in the Minkowski space already describe a family of spiral (circular, in particular,) orbits for a spinning particle in addition to linear motions $[15,16]$. The physical reason for such solutions to appear was established by Möller [17]: in special relativity, in distinction from the Newtonian mechanics, the center of mass of a rotating body is located depending on the reference frame. Condition (3) separates the motion of a set of centres of mass rather than of a single center of mass. In this case the linear motion is executed by the proper center of mass (according to the terminology of [18]; it is the closest analog of the Newtonian center of mass) and the axis of rotation, while the unproper centers of mass engage in the spiral motion. All the centers of mass are inside the geometric size of a convex body [18]. 
To eliminate the second derivatives from the velocity in the MPE, instead of equations (1) or (4), the equation

$$
M \frac{\mathrm{D} u^{\lambda}}{\mathrm{d} s}=-\frac{1}{2} u^{\pi} S^{\rho \sigma} R_{\pi \rho \sigma}^{\lambda}
$$

was often considered. It means that for the analytic in spin solutions of the MPE only linear in spin terms of these equations were taken into account. However, it is necessary to have the estimations of the neglected terms in equation (1) for any solution of the exact MPE, too. We have investigated the values of these terms in the case of equatorial motions of a spinning test particle in the Schwarzschild gravitational field [19]. For this purpose we used the known first integrals of the MPE, energy and angular momentum, in the standard Schwarzschild coordinates $x^{1}=r, \quad x^{2}=\theta, \quad x^{3}=\varphi, \quad x^{4}=t($ at $\theta=\pi / 2)[20]$. It is shown that for any solution of the exact MPE (1)-(3) with fixed initial values of coordinates, velocity, and a spin of a particle (i.e. both for the solution describing the motion of the proper center of mass and the solutions of unproper centers), the neglect of the indicated terms of equation (1) leads to a relative error, in expressions for the energy or the initial acceleration of a particle, no less than $1 / 3$ if

$$
u_{\perp 0}^{2}>\frac{M r_{0}}{3\left|S_{0}\right|}\left(1-\frac{2 m}{r_{0}}\right)^{-1 / 2}
$$

and $\left(u_{\| 0} / u_{\perp 0}\right)^{2} \ll 1[19]$, p. 30. Here $u_{\perp 0}, u_{\| 0}$ are initial values of the tangential and radial components of the 4 -velocity; $S_{0}$ is the spin constant; $m$ is the mass of the gravitational field source; $r_{0}$ is the initial value of the radial coordinate. By the test condition for a spinning particle [5]

$$
\frac{\left|S_{0}\right|}{M r} \ll 1
$$

in (6) we have $u_{\perp 0}^{2} \gg 1$, that is, the tangential component of the 4-velocity is ultrarelativistic. Such velocity corresponds to a large value of the particle orbital angular momentum.

So, the criterion of the increase of the gravitational spin-orbit interaction (6) determines sufficient conditions under which the influence of this interaction becomes so large that for its correct estimation it is necessary to go beyond the linear in spin approximation of the MPE. One can call (6) a mathematical criterion because it was obtained as the result of the formal comparison of equations (1) and (5) solutions. However, the criterion (6) has a physical meaning, too, and we will consider it below.

\section{The energy criterion}

Let us consider the energy of a spinning test particle for such equatorial motions in the Schwarzschild field which can be described by the analytic in spin solutions 
of the MPE. (So, we exclude from the consideration the motions of unproper centers of mass). Then, within the accuracy of the quadratic in spin term we have $[21]$

$$
E=M u_{4}\left[1-\frac{m}{r} \frac{u_{\perp}}{u_{4}} \frac{S_{0}}{M r}-\frac{3 m}{r} u_{\perp}^{2}\left(\frac{S_{0}}{M r}\right)^{2}\right]
$$

where

$$
u_{4}=\left[u_{\|}^{2}+\left(1-\frac{2 m}{r}\right)\left(1+u_{\perp}^{2}\right)\right]^{1 / 2} .
$$

Factor $M u_{4}$ in (8) is the known value of energy for geodesic motions. Both the linear in spin term and the square in spin term of (8) have the spin-orbital nature, both are not equal to 0 , only if $u_{\perp} \neq 0$. The absolute values of the two terms increase when $\left|u_{\perp}\right|$ is increasing. However, coefficient $m u_{\perp} / r u_{4}$ near the small quantity $S_{0} / M r$ (see (7)) is not more than $1 / 2$ for all the values of $u_{\perp}$ (we put $r>2 m$, i.e. consider the region on the outside of the horizon surface). Whereas coefficient $3 m u_{\perp}^{2} / r$ near $\left(S_{0} / M r\right)^{2}$ becomes equal to a large value if $u_{\perp}^{2}$ is sufficiently large. Therefore, we cannot be sure that the linear in spin term in (8) gives a good approximation for the energy, regardless of the value of the particle's ultrarelativistic orbital velocity. (Unfortunately, as usual, such a restricted analysis was the last point of the investigation on this problem, see, e.g., [22]). Indeed, we note that the absolute value of the quadratic in spin term of (8) is larger than that of the linear in spin term if

$$
\left|u_{\perp}\right| u_{4}>\frac{M r}{3\left|S_{0}\right|}
$$

Obviously, the quadratic in spin term of (8), as well as any finite number of terms with spins in the series for the energy, cannot ensure a correct approximation for the energy in the whole range of the tangential velocity, including $u_{\perp}^{2} \gg 1$. Nevertheless, one can consider (9) as a criterion of the increase of the gravitational spin-orbit interaction. It is easy to see that in the case $\left(u_{\perp} / u_{\|}\right)^{2} \gg 1$ the criterion (9) is close to (6).

\section{The force criterion}

The absolute value of the spin-orbit force acting on a spinning test particle in the Schwarzschild field from the viewpoint of a comoving observer is $[3,10,11,21]$

$$
|\vec{F}|=\frac{m M}{r^{2}} \frac{3\left|S_{0} u_{\perp}\right|}{M r} \sqrt{1+u_{\perp}^{2}}
$$

(for clarity, we separated in (10) the Newtonian gravitational force $m M / r^{2}$ and small quantity (7); equation (10) is written for equatorial motions only, the corresponding generalization for any motions see in [23]). Exactly, force (10) deflects the spinning particle motion from the geodesic free fall and is used in [10] for the analysis of the weak equivalence principle. One may consider (10) as a generalization of equation (44) from [5] for any velocities of a spinning particle. This 
generalization is not trivial and contains significant information on the gravitational spin-orbit interaction. Namely, when $\left|u_{\perp}\right| \ll 1$ according to (10) and (7), we have $|\vec{F}| \ll m M / r^{2}$, whereas in the ultrarelativistic region of velocities, when $\left|u_{\perp}\right| \gg 1,|\vec{F}| \gg m M / r^{2}$. In particular, we can formulate such a criterion of the increase of the gravitational spin-orbit interaction:

$$
\left|u_{\perp}\right| \sqrt{1+u_{\perp}^{2}}>\frac{M r}{3\left|S_{0}\right|}
$$

In accordance with (10), when non-equality (11) is fulfilled, the spin-orbit force for a comoving observer is larger than the Newtonian force.

\section{Conclusion}

Although the three criteria of the increase of the gravitational spin-orbit interaction (6), (9), (11) have different meanings, they are almost the same quantitatively if $\left(u_{\perp} / u_{\|}\right)^{2} \gg 1$. As in this case $\left|u_{\perp}\right|$ is approximately equal to the relativistic Lorentz $\gamma$ - factor for a spinning test particle, all these criteria can be approximately written in the form:

$$
\gamma \approx\left|u_{\perp}\right|>\sqrt{\frac{M r}{3\left|S_{0}\right|}} .
$$

So, if the $\gamma$ - factor of an ultrarelativistic particle has an order of the small quantity (7) to the power $-1 / 2$, the gravitational spin-orbit interaction cannot be neglected. For example, without taking into account this interaction it is impossible to construct a strict theory of black holes. (Now theoretical conclusions concerning black holes are based on the analysis of geodesic world lines only). It is important that the partial solutions of the MPE in the Schwarzschild and Kerr fields demonstrate that the values of the $\gamma$ - factor (12) are sufficient for the realization of essentially nongeodesic circular orbits of a spinning test particle near the source of a gravitational field [19,24]. More exactly, tangential velocity on these orbits has the same order as the right-hand side of (12).

Obviously, natural macroscopic bodies have too small velocities for their spinorbit interaction to be significant; but it is not the case with elementary particles. In particular, we obtain from (12) that an electron in its path near the Earth's surface acquires an additional nongeodesic acceleration (from the viewpoint of a comoving observer) which leads to the Newtonian acceleration of the free fall $9.8 \mathrm{~m} / \mathrm{s}^{2}$ if the electron's ultrarelativistic velocity corresponds to its free motion energy of the order of $10^{15} \mathrm{eV}$, the value typical of cosmic rays. Another example: criterion (6) is fulfilled for an electron near the surface of a neutron star with the mass approximately equal to the mass of the Sun if the corresponding energy is equal to $10^{14} \mathrm{eV}$.

So, the role of the gravitational ultrarelativistic spin-orbit interaction in the behaviour of elementary particles in strong gravitational fields is to be investigated. 


\section{References}

1. Mathisson M. Neue mechanik materieller systeme. // Acta Phys. Pol., 1937, bd. 6, s. $163-200$.

2. Papapetrou A. Spinning test-particles in general relativity. // Proc. Roy. Soc. A, 1951, vol. 209, p. 248-258.

3. Plyatsko R.M. Sixty years of investigation of the Mathisson-Papapetrou equations. // Ukr. Fiz. Zhurn., 1997, vol. 42, No 7, p. 778-784 (in Ukrainian).

4. Corinaldesi E., Papapetrou A. Spinning test-particle in general relativity. 2. // Proc. Roy. Soc. A, 1951, vol. 209, p. 259-268.

5. Wald R. Gravitational spin interaction. // Phys. Rev. D, 1972, vol. 6, No 2, p. 406-413.

6. Gravitation and relativity (Edited by Hong-Yee Chiu and W.F.Hoffmann). New York - Amsterdam, W.A. Benjamin INC, 1964.

7. Misner C.W., Thorne K.S., Wheeler J.A. Gravitation. San Francisco, Freeman, 1973.

8. Rüdiger R. The Dirac equation and spinning particles in general relativity. // Proc. Roy. Soc. A, 1981, vol. 377, p. 417-424.

9. Will C.M. Theory and experiment in gravitational physics. Cambridge, Cambridge University Press, 1981.

10. Plyatsko R.M. On the weak equivalence principle. // Ukr. Fiz. Zhurn., 1996, vol. 41, No 2, p. 133-137 (in Ukrainian).

11. Plyatsko R.M. New aspects of gravitational interaction. // Collected Physical Papers of Shevchenko Scientific Society, 1996, vol. 2, p. 297-307 (in Ukrainian).

12. Wienberg S. Gravitation and cosmology: principles and applications of general relativity. New York, Wiley, 1972.

13. Pirani F. On the physical significance of the Riemann tensor. // Acta Phys. Pol., 1956, vol. 15, No 6, p. 389-405.

14. Mashhon B. Particles with spin in a gravitational field. // J. Math. Phys., 1971, vol. 12, No 7, p. 1075-1077.

15. Mathisson M. Das zitternde electron und seine dynamic. // Acta Phys. Pol., 1937, bd. 6 , s. $218-227$.

16. Weyssenhoff J., Raabe A. Relativistic dynamics of spin-fluid and spin-particles. // Ibid, 1947, vol. 9, No 1, p. 7-18.

17. Möller C. On the definition of the center of gravity of an arbitrary closed system in the theory of relativity. // Commun. Dublin Inst. Advan. Stud. Ser. A, 1949, No 5, p. $3-19$.

18. Möller C. The theory of relativity. Oxford, Oxford University Press, 1972.

19. Plyatsko R.M. Manifestations of gravitational ultrarelativistic spin-orbit interaction. Kyiv, Naukova Dumka, 1988 (in Ukrainian).

20. Micoulaut R. Über die Bewegungsgleichungen eines makroskopischen rotierenden Teilchens gemäb der allgemeinen Relativitätstheorie. // Z. Phys., 1967, bd. 206, s. 394403.

21. Plyatsko R.M. Classical spinning particle in a gravitational field. // Kinem. and Phys. Celest. Bodies, 1993, vol. 9, No 5, p. 65-72.

22. Rasband S. Black holes and spinning test bodies. // Phys. Rev. Lett., 1973, vol. 30, No 3, p. 111-114.

23. Plyatsko R.M. Gravitational spin-orbit force for any velocities and spin orientation. // Ukr. Fiz. Zhurn., 1997, vol. 42, No 4, p. 389-394 (in Ukrainian). 
24. Plyatsko R.M., Vynar A.L. Essentially nongeodesic motions of a rotating test body in the general theory of relativity. // Sov. Phys. - Dokl. (Engl. transl.), 1982, vol. 24, No 4, p. $328-330$.

\section{Ґравітаційна спін-орбітальна взаємодія: критерії росту}

\section{Р.Пляцко}

Інститут прикладних проблем механіки і математики

ім. Я.С.Підстригача НАН України, 290601 м. Львів, вул. Наукова, $3^{5}$

Отримано 10 березня 1998 р.

На основі рівнянь Матісона-Папапетру досліджено ґравітаційну спінорбітальну взаємодію в ультрарелятивістичному діапазоні орбітальної швидкості пробної частинки зі спіном у полі Шварцшільда. Проаналізовано різні ознаки суттєвого зростання цієї взаємодії, коли танґенціяльна швидкість стає ультрарелятивістичною. Основним фактором росту є квадрат релятивістичного $\gamma$-фактора Лоренца. Наведено числові оцінки для високоенергетичного електрона біля поверхні Землі і нейтронної зорі. Підкреслено, що широко розповсюджене припущення про припливні сили як причину відхилення пробної частинки зі спіном від геодезійної траєкторії $€$ некоректним. Справжньою причиною такого відхилення $є$ ґравітаційна спін-орбітальна взаємодія.

Ключові слова: рівняння Матісона-Папапетру, ультрарелятивістичний рух

PACS: 04.20. $-q$, 95.30.Sf, 98.80.Dr 